\title{
Dynamics of point Josephson junctions in a microstrip line
}

\author{
J.G. Caputo and L. Loukitch \\ Laboratoire de Mathématiques, INSA de Rouen \\ B.P. 8, 76131 Mont-Saint-Aignan cedex, France
}

June 25, 2018

\begin{abstract}
We model the dynamics of point Josephson junctions in a $1 \mathrm{D}$ microstrip line using a wave equation with delta distributed sine nonlinearities. The model is suitable for both low $\mathrm{T}_{c}$ and high $\mathrm{T}_{c}$ systems $(0$ and $\pi$ junctions). For a single junction in the line, we found two limiting behaviors: the ohmic mode where the junction acts as a pure resistor which stops waves and separates the cavity and the junction mode where the wave is homogeneous throughout the strip. This classification allows to bound the IV curves of the system. Two junctions in a strip give generally ohmic modes and combined junction/ohmic modes and yield information about the behavior with an array with many junctions. Finally we use this analysis to understand the many junction case for 0 and $\pi$ junctions and the effect of an external magnetic field.
\end{abstract}

\section{Introduction}

Type I Superconductors are characterized by the phase of a complex number, the macroscopic order parameter. Josephson [1] showed that when two such superconductors are very close, electrons can tunnel from one to the other according to the relations

$$
V=\frac{\Phi_{0}}{2 \pi} \frac{d \phi}{d t}, I=s J_{c} \sin (\phi),
$$


where $V$ and $I$ are respectively the voltage and current across the barrier, $\phi$ is the difference between the phases in the two superconductors, $s$ is the contact surface, $J_{c}$ is the critical current density and $\Phi_{0}$ is the flux quantum. The two Josephson relations together with Maxwell's equations imply the modulation of DC current by an external magnetic field in the static regime (SQUIDs) and the conversion of $\mathrm{AC}$ current into microwave radiation.

The ratio of the electromagnetic flux to the flux quantum gives the Josephson length, the typical distance of variation of the phase $\lambda_{J}=\frac{\Phi_{0}}{L J_{c}}$. Depending whether their length $l$ is larger or not than $\lambda_{J}$, Josephson junctions exhibit significantly different behaviors. Small junctions for which $l<\lambda_{J}$ are used as SQUIDS to measure magnetic fields in the static regime or as signal mixers where the steep IV characteristic at the gap is used to downshift an unknown high frequency signal by combining it with a known local oscillator signal. In this latter context Salez et al 4] suggested to use several unequally spaced junctions, instead of just one, to get a better frequency response over a large frequency band. Long Josephson junctions for which $l>>\lambda_{J}$ are described by the $1 \mathrm{D}$ sine-Gordon equation

$$
\phi_{t t}-\phi_{x x}+\sin (\phi)=0,
$$

which admit the kink solutions varying from 0 to $2 \pi$. Long junctions have a characteristic dependence of the maximum critical current in the presence of magnetic field because of the screening of the applied field by the supercurrent. In the dynamical regime long junctions are used as microwave generators but provide small output power and poor impedance matching to the transmission line. To improve on this, series arrays of junctions have been used, with success since they have been shown to synchronize in the presence of external forcing [15] [16] giving rise to an $N^{2}$ power output for $N$ junctions. Such behavior has been understood both from the quantum point of view [12] and the Resistive Shunted Junction (RSJ) equivalent circuit model [13]. The cavity surrounding the junctions stores radiation which can increase the output power of the device.

The mathematical description of such a system is a wave equation for the cavity coupled to the $2 \mathrm{D}$ version of the sine-Gordon equation (2) via interface conditions. In [5, 6] one of the authors studied this coupling for a long junction with a small surrounding passive region. When the cavity is present only at the junction tip and the miss-match at the interface is not too large, the kink 
crosses it unaffected and adapts its speed. When the passive region surrounds the junction, it sets the kink speed. This ballistic kink motion will be absent for small junctions so in the present work we consider the coupling of a large cavity to one or a few small junctions. We want to understand how junctions can talk to each other via the surrounding passive region. Two approximations are assumed, we consider the 1D limit where transverse modes of the microstrip do not play a role and neglect the phase variation inside the junctions, in other words we neglect the junction length. This is valid for an array of short junctions whose length remains smaller than the wave length of the radiation.

This approach can be applied to high Tc superconductors where due to a different symmetry of the wave function of the Cooper pairs one has current phase relations that involve the higher harmonics 7 . This different symmetry can also induce a negative coupling in (10) leading to so-called $\pi$ junctions. Normal (0) and $\pi$ junctions can be associated to form $0-\pi$ junctions which have semifluxon solutions that vary between 0 and $\pi$ and are attached to the discontinuity. In the static regime the maximum DC current going through the system is strongly modulated for a short junction like for a SQUID while it is constant for small field when the junction is longer [8]. The IV characteristics give resonances that are similar to zero field steps [8, 9. Note however the difference between the ballistic motion of a kink in a (0) long Josephson junction and the hopping of semi-fluxons in a $(0-\pi)$ junction. As for 0 junctions, $(0-\pi)$ junctions can be organized as arrays [10, 11] and these have interesting paramagnetic behavior.

The model we introduce allows to describe accurately the coupling between small junctions in such an array. Both 0 and $\pi$ junctions can be considered and disorder can be added. For simplicity we have considered the large damping limit where it is possible to develop the analysis. We studied in detail the cases of one and two junctions that remain fairly simple and used our conclusions for the case of many junctions. For a single junction, we found two limiting behaviors: the ohmic mode where the junction acts as a pure resistor which stops waves and separates the cavity and the junction mode where the wave is homogeneous throughout the microstrip. After introducing the model is section 2 , we consider these limiting behaviors in sections 3,4 and 5. These allow to understand in detail the features of the IV characteristics (section 6). The behavior of two junctions shown in section 7 follows generally ohmic modes 
and combined junction/ohmic modes. Using this, in section 8 we generalize the study to many junctions embedded in a microstrip and understand the influence of the sign of the current density ( 0 or $\pi$ junctions) and external magnetic field. Conclusions are presented in section 9 .

\section{The model}

The device we model shown in the top panels of Fig. 1](top view on the left and section on the right) is a narrow microstrip in which are embedded a number of small junctions in the window design. We proceed from the description of the two superconductors as two inductance arrays connected by capacitive elements (resp. and a resistor and nonlinear Josephson element) in the passive (resp. junction) region. The equivalent circuit is shown in the bottom panel of Fig. 1] In the following subsections we show that the continuum limit yields an inhomogeneous 2D sine-Gordon equation which we reduce to $1 \mathrm{D}$ when the microstrip is narrow. For small junctions we then justify our delta function approach (20).

\subsection{RSJ model for distributed Josephson junctions}

A simple mathematical model of the window Josephson junction is to describe each superconductor by an array of inductances $L$. The coupling elements between two adjacent nodes in each array are, a capacitor $C$, resistance $R$ and Josephson current $I_{c}$ [2]. The Kirchoff laws at each couple of nodes $\left(i_{b}, i_{t}\right)$ in the bottom and top superconducting layers can be combined to give the relation expressing the conservation of currents at node $i$ in the device

$$
C_{i} \ddot{\Phi}_{i}+\sum_{j} \frac{\Phi_{i}-\Phi_{j}}{L}+I_{i}^{c} \sin \frac{\Phi_{i}}{\Phi_{0}}+\frac{\dot{\Phi}_{i}}{R_{i}}=0,
$$

where $\Phi=\Phi_{t}-\Phi_{b}$ (resp. $\Psi=\Psi_{t}-\Psi_{b}$ ), is the phase difference between the two superconductors in the junction (resp. passive) part, the summation $\sum_{j}$ is applied to the nearest neighbors, $I_{i}^{c}$ is the critical current which is non zero only in the junctions and $R_{i}$ is the resistance associated to the current of quasiparticles again non zero only in the junctions. In agreement with experiments, we have assumed the same surface inductance for the whole sample but have 
taken into account the variation of the capacity $C$ and resistance $R$ from the junctions to the rest of the cavity. Note that equation (3) is a discretisation of Maxwell's equations (wave equation part) and Josephson constitutive equations (sine term), assuming an electric field normal to the plates, a magnetic field in the junction plane and perfect symmetry between the top and bottom superconducting layers. We can now obtain the model in the continuum limit, more suitable for analysis.

\subsection{Continuum limit}

The continuum version of the system (3) can be derived by introducing the following quantities per unit area $\left(a^{2}\right)$ of elementary cells of length $a$.

$$
\overline{C_{J}}=\frac{C_{J}}{a^{2}}, \quad j_{i}^{c}=\frac{I_{i}^{c}}{a^{2}}, \quad \overline{r_{i}}=R_{i} a^{2} .
$$

We normalize the phases by the flux quantum $\Phi_{0}$,

$$
\varphi_{i}=\frac{\Phi_{i}}{\Phi_{0}}
$$

and introduce the Josephson characteristic length

$$
\lambda_{J}^{2}=\frac{\Phi_{0}}{j_{c} L_{J}} .
$$

Notice that in this 2D problem the inductance associated to each cell is equal to the branch inductance $L$. To differentiate the junction regions from the passive regions we introduce the indicator function $g_{i}$ such that $g_{i}=1$ in the junctions and 0 elsewhere. We can then write

$$
j_{i}^{c}=g_{i} j^{c}, \frac{1}{\overline{r_{i}}}=g_{i} \frac{1}{\bar{r}} \text { and } \bar{C}_{i}=g_{i} \bar{C}_{J}+\left(1-g_{i}\right) \bar{C}_{I}
$$

where $j^{c}, \bar{r}, \bar{C}_{J}$ and $\bar{C}_{I}$ are respectively the critical current densities, the quasi particle resistance in the junction, the capacitance per unit area in the junction and the capacitance per unit area in the passive region.

We substitute relations (4) - (7) in (3) and obtain

$$
L \bar{C}_{I} \ddot{\varphi}_{i}+\sum_{j} \frac{\varphi_{i}-\varphi_{j}}{a^{2}}+g_{i}\left[L\left(\bar{C}_{J}-\bar{C}_{I}\right) \ddot{\varphi}_{i}+\frac{1}{\lambda_{J}^{2}} \sin \varphi_{i}+\frac{L}{\bar{r}} \dot{\varphi}_{i}\right]=0,
$$

which in the continuum limit yields

$$
L \bar{C}_{I} \varphi_{t t}-\Delta \varphi+\frac{1}{\lambda_{J}^{2}} g(x, y)\left[L \lambda_{J}^{2}\left(\bar{C}_{J}-\bar{C}_{I}\right) \varphi_{t t}+\sin \varphi+\frac{L \lambda_{J}^{2}}{\bar{r}} \varphi_{t}\right]=0 .
$$


To obtain the final equation we rescale time by the inverse of the plasma frequency $\omega_{I}^{-1}=\lambda_{J} \sqrt{L \bar{C}_{I}}$ so that $\tilde{t}=\omega_{I} t$. As unit of space we use the Josephson length, $\tilde{x}=x / \lambda_{J}, \tilde{y}=y / \lambda_{J}$. The final equation is then

$$
\varphi_{t t}-\Delta \varphi+g(x, y)\left(c \varphi_{t t}+\sin \varphi+\alpha \varphi_{t}\right)=0,
$$

where the tildes have been omitted for simplicity and where the coefficients $c$ and $\alpha$ are

$$
c=\frac{\bar{C}_{J}}{\bar{C}_{I}}-1, \quad \alpha=\frac{\lambda_{J}}{\bar{r}} \sqrt{\frac{L}{\bar{C}_{I}}}
$$

The boundary conditions represent the input of an external current $I$ or magnetic field $b$ on the device. They are

$\left.\varphi_{x}\right|_{x=0}=b-(1-\nu) \frac{I}{2 w},\left.\varphi_{x}\right|_{x=l}=b+(1-\nu) \frac{I}{2 w},\left.\varphi_{y}\right|_{y=0}=-\nu \frac{I}{2 l},\left.\varphi_{y}\right|_{y=w}=\nu \frac{I}{2 l}$,

where $0<\nu<1$. The case $\nu=1$ corresponds to a pure inline current feed and $\nu=0$ to a pure overlap current feed. We will mostly consider the latter case in the discussion.

\subsection{Reduction to a 1D problem}

When the microstrip is narrow and the junctions occupy the whole width we can assume that $g$ is independent of $y$. We can then write

$$
\varphi(x, y, t)=\nu I \frac{y^{2}}{2 s}+\sum_{n=0}^{\infty} \phi_{n}(x, t) \cos \left(\frac{n \pi y}{w}\right),
$$

where the first term takes care of the current feed and the second satisfies homogeneous Neumann boundary conditions for $y=0, w$.

The calculations detailed in [5] show that for a narrow width and small current only the first mode $\phi_{0}$ needs to be taken into account. We then obtain the following equation for $\phi_{0}$ where the 0 has been dropped for simplicity

$$
\phi_{t t}-\phi_{x x}+g(x)\left(c \phi_{t t}+\sin \phi+\alpha \phi_{t}\right)=\nu \frac{I}{s},
$$

with the boundary conditions $\phi_{x_{x=0}}=-(1-\nu) \frac{I l}{2 s} \quad \phi_{x_{x=l}}=(1-\nu) \frac{I l}{2 s}$. In the following we will write

$$
j=\frac{I}{s},
$$

and assume an overlap current feed $(\nu=1)$ except in section 7 . 


\subsection{The delta function model}

As the area of the junction is reduced the total super-current is reduced and tends to zero. To describe situations where the area is small so that the variations of the phase can be neglected in the junction but the supercurrent is significant we introduce the following delta function model. Introduce the function $g(x)=d_{a} g_{h}(x)$

$$
\begin{aligned}
g_{h}(x)=\left\{\begin{array}{rr}
1 /(2 h) & a-h<x<a+h \\
0 & \text { elsewhere }
\end{array}\right. \\
\phi_{t t}-\phi_{x x}+d_{a} g_{h}(x)\left(\sin (\phi)+\alpha \phi_{t}+c \phi_{t t}\right)=j .
\end{aligned}
$$

In the following we assume $\phi$ to be $C^{2}$ in $x$ and $t$ and $\phi_{t t x}$ to be continuous. We now show that equation (16) converges when $h \rightarrow 0$ to

$$
\phi_{t t}-\phi_{x x}+d_{a} \delta(x-a)\left(\sin (\phi)+\alpha \phi_{t}+c \phi_{t t}\right)=j .
$$

For that introduce the Taylor expansion of $\phi$ near $x=a$

$$
\phi(x, t)=\phi(a, t)+(x-a) \phi_{x}(a, t)+O\left((x-a)^{2}\right) .
$$

In the following for simplicity of writing we will omit the $t$ and $x$ dependencies. We integrate (16) from $a-h$ to $a+h$ (see details in appendix: Continuum limit). The first term

$$
\int_{a-h}^{a+h} \phi_{t t} d x \rightarrow O \text { when } h \rightarrow 0
$$

the second one

$$
\int_{a-h}^{a+h} \phi_{x x} d x=\phi_{x}(a+h)-\phi_{x}(a)
$$

and the third one

$$
\lim _{h \rightarrow 0} \frac{d_{a}}{2 h} \int_{a-h}^{a+h}\left(\sin (\phi)+\alpha \phi_{t}+c \phi_{t t}\right) d x=d_{a}\left(\sin (\phi(a))+\alpha \phi_{t}(a)+c \phi_{t t}(a)\right),
$$

when $h \rightarrow 0$ which is consistent with the model

$$
\phi_{t t}-\phi_{x x}+d_{a} \delta(x-a)\left(\sin (\phi)+\alpha \phi_{t}+c \phi_{t t}\right)=j,
$$

together with the boundary conditions $\phi_{x}(x=0)=0, \quad \phi_{x}(x=l, t)=0$. 
In the following we will consider the case $c=0$ for simplicity. We assume the same capacity in the junction and in the passive region so that the final equation is

$$
\phi_{t t}-\phi_{x x}+d_{a} \delta(x-a)\left(\sin (\phi)+\alpha \phi_{t}\right)=j .
$$

This equation can be considered as a 1D model of a point Josephson junction embedded in a microstrip cavity.

\section{The single junction case}

The partial differential equation (20) involves a distribution, however its solution is differentiable except at the junction points. To solve it numerically we integrate the equation over reference intervals using the finite volume approach. The resulting system of ordinary differential equations is then solved using the DOPRI5 4th and 5th order Runge-Kutta variable step integrator developed by Hairer and Norsett [17]. Details of the numerical code are given in the second part of the appendix.

We now consider the two limiting cases of a static solution and high voltage for which simple expressions of the solution can be obtained. In particular these have been used to validate our numerical procedure.

\subsection{Static behavior}

We assume $\phi_{t}=\phi_{t t} \equiv 0$ in (20) and get

$$
\begin{aligned}
-\phi_{x x}+\delta(x-a) d_{a} \sin (\phi) & =j \\
\left.\phi_{x}\right|_{x=0, l} & =0
\end{aligned}
$$

For $x \neq a,-\phi_{x x}=j$ so that for $x<a, \phi=\phi_{l} \equiv-\frac{j}{2} x^{2}+C_{l} x+D_{l}$ and for

$x>a, \phi=\phi_{r} \equiv-\frac{j}{2} x^{2}+C_{r} x+D_{r}$. Integrating (21) over the whole domain we get the conservation of current

$$
d_{a} \sin (\phi(a))=j l,
$$

so that no solution exists for $|j l|>d_{a}$.

The solution is obtained as usual by assuming continuity of the phase and using the jump condition on the gradient obtained by integrating on a small 
domain including the junction

$$
\left[\phi_{x}\right]_{a^{-}}^{a^{+}}=d_{a} \sin (\phi(a))
$$

Using these two constraints we obtain the static solution

$$
\phi_{s}(x)= \begin{cases}\frac{j}{2}(a-x)(a+x)+\arcsin \left(\frac{j l}{d_{a}}\right) & x<a \\ \frac{j}{2}\left((a-x)(x+a+2 l)+\arcsin \left(\frac{j l}{d_{a}}\right)\right. & a<x\end{cases}
$$

\subsection{High voltage behavior}

Since the Josephson current is bounded, its influence in (20) will decrease as the current $j$ is increased. In this limit of high current the phase is growing very fast so that $\phi_{t}$ is close to the average voltage $V \equiv\left\langle\phi_{t}\right\rangle_{t}$. We can write approximately $\phi(x, t)=V t+\phi_{v}(x)$ so that we can average 20 assuming $\left\langle\phi_{t}(x, t)\right\rangle_{t}=V$, $\langle\sin (\phi(a))\rangle_{t}=0$ and $\left\langle\phi_{t t}\right\rangle_{t}=0$, yielding

$$
-\phi_{v} x x+\delta(x-a) d_{a} \alpha V=j
$$

with the boundary conditions $\left.\phi_{v_{x}}\right|_{x=0, l}=0$. Integrating this equation over the whole domain we get $V=\frac{j l}{d_{a} \alpha}$.

Using the same constraints as in the static case (continuity of the phase and jump of its gradient at the junction) we get up to a constant the high voltage solution $\phi_{v}$

$$
\phi_{v}(x, t)= \begin{cases}\frac{j}{2}(a-x)(a+x)+V t+C & x<a \\ \frac{j}{2}(a-x)(a+x-2 l)+V t+C & a<x\end{cases}
$$

Notice that this and precedent solutions have the same spatial behavior; $\phi_{s}-$ $\phi_{v}=V t+C$. We will not find this result with more junctions (except in particulary cases).

\subsection{Fourier representation}

Because of the homogeneous Neumann boundary conditions one can write the solution as a cosine Fourier series

$$
\phi(x, t)=\sum_{n=0}^{+\infty} A_{n}(t) \cos \left(\frac{n \pi x}{l}\right) .
$$


The solution of (20) has a discontinuous first spatial derivative to that the amplitude of the mode $A_{n}$ decreases as $1 / n^{2}[18$. This means that in general many modes are needed for the description of the solution. However since the system is close to a linear one we will see that these modes provide insight into the limiting behaviors.

Plugging (28) into (20) and projecting we get the evolution of the modes,

$$
\begin{gathered}
l A_{0 t t}+d_{a}\left(\sin \left(\phi_{a}\right)+\alpha \phi_{a t}\right)-j l=0, \\
A_{n t t}+\left(\frac{n \pi}{l}\right)^{2} A_{n}+\frac{2 d_{a}}{l} c_{n}\left(\sin \left(\phi_{a}\right)+\alpha \phi_{a t}\right)=0,
\end{gathered}
$$

where we have introduced the coupling coefficients associated to the junction at $x=a, c_{n}=\cos \left(\frac{n \pi a}{l}\right)$. Equations (29) and (30) are coupled through the term $\sin \left(\phi_{a}\right)+\alpha \phi_{a t}$. Due to this particular feature, two limiting behaviors can be expected.

- The ohmic mode for which

$$
\left.d_{a} \alpha \phi_{a}\right|_{t}-j l=0
$$

Then the equations for the $A_{n}$ have a right hand side so that the cavity modes are driven by the junction.

- The junction mode such that

$$
d_{a}\left(\sin \left(\phi_{a}\right)+\alpha \phi_{a t}\right)-j l=0 .
$$

In this case the equations for the $A_{n}$ have a constant right hand side $(=j l)$. We will show that in this case the cavity is synchronized with the junction.

The equations giving the evolution of $\phi_{a}$ in these two limiting behaviors can be solved yielding for the ohmic mode

$$
\phi_{a}=\frac{j l}{d_{a} \alpha} t+C_{1}
$$

where $C_{1}$ is a constant and a voltage

$$
V_{o}=\frac{j l}{d_{a} \alpha}
$$


For the junction mode the equation can be solved using separation of variables and we get

$$
\phi_{a}=2 \arctan \left\{\frac{d_{a}}{j I}\left[\alpha V_{j} \tan \left(\frac{V_{j}}{2}\left(t+C_{2}\right)\right)+1\right]\right\},
$$

where the junction mode voltage $V_{j}$ is

$$
V_{j}=\frac{1}{\alpha} \sqrt{\left(\frac{j l}{d_{a}}\right)^{2}-1}
$$

where $C_{2}$ is a constant.

Fig. [2] shows these two limiting behaviors for a junction placed in $a=-1.57$ with $d_{a}=1 l=10$ and a current $j \approx 0.1374$. The numerical solution is presented with the crosses while the analytical estimates (33) and (35) are shown in continuous line. The left panel corresponds to the ohmic mode and one can see the excellent agreement of the numerical solution with the line given by (33). The right panel presents the junction mode and the extension of the estimate (see below), again the agreement is very good. Notice that for the same time interval the variation of $\phi_{t}(a, t)$ is different for the two panels of Fig. 2 so that we will get a different average voltage for these two behaviors.

\section{The ohmic mode}

In this case $\phi_{t}(a, t)=V_{o}=\frac{j l}{\alpha d_{a}}$. To get the field we need to solve on each subdomain $[0, a[$ and $] a, l]$ the following problems

$$
\left\{\begin{array} { l } 
{ \phi _ { t t } - \phi _ { x x } = j } \\
{ \phi _ { x } | _ { 0 } = 0 , \quad \phi _ { t } | _ { a } = \frac { j l } { \alpha d _ { a } } }
\end{array} \quad \left\{\begin{array}{l}
\phi_{t t}-\phi_{x x}=j \\
\left.\phi_{t}\right|_{a}=\frac{j l}{\alpha d_{a}},\left.\phi_{x}\right|_{l}=0 .
\end{array}\right.\right.
$$

Consider the left subdomain $\left[0, a\left[\right.\right.$, we introduce $\phi(x, t)=v(x, t)+\frac{j l t}{\alpha d_{a}}$ so that if $\phi$ solves the left problem then $v$ solves

$$
\begin{array}{r}
v_{t t}-v_{x x}=j \\
\left.v_{x}\right|_{0}=\left.0 \quad v_{t}\right|_{a}=0
\end{array}
$$

A particular solution of (37) is $v_{l}=-\frac{j}{2} x^{2}+D_{l}$ where $D_{l}$ is an integration constant. Then $w=v-v_{l}$ solves the homogeneous wave equation

$$
\partial_{t t} w-\partial_{x x} w=0,
$$


with $\left.w_{x}\right|_{0}=0$ and $\left.w_{t}\right|_{a}=0$. We can assume without loss of generality that $w(x, 0)=0$. The condition $\left.w_{x}\right|_{0}=0$ imposes a solution of the type

$$
w(x, t)=a_{l} \sin \left(V_{l} t\right) \cos \left(V_{l} x\right),
$$

where $V_{l}$ is a constant. Also, $\left.w_{t}\right|_{a}=0$ imposes a condition on $V_{l}$ because

$$
w_{t}(x=a, t)=a_{l} \sin \left(V_{l} t\right) \cos \left(V_{l} a\right)=0
$$

is possible for all $t$ 's only if $\cos \left(V_{l} a\right)=0$. So that we get

$$
V_{l}=\frac{2 k_{l}+1}{2} \frac{\pi}{a}
$$

The problem is identical for $x \in] a, l]$ and using similar arguments we get $v_{r}=$ $-\frac{j}{2}(x-l)^{2}+D_{r}$ and

$$
V_{r}=\frac{2 k_{r}+1}{2} \frac{\pi}{a-l}
$$

We obtain a solution for the equation (20). The phase on the whole domain is then

$$
\phi(x, t)= \begin{cases}\frac{j l t}{\alpha d_{a}}+a_{l} \sin \left(V_{l} t\right) \cos \left(V_{l} x\right)+\frac{j}{2}(a-x)(a+x) & x<a \\ \frac{j l t}{\alpha d_{a}}+a_{r} \sin \left(V_{r} t\right) \cos \left(V_{r}(x-l)\right)+\frac{j}{2}(a-x)(a+x-2 l) & a<x\end{cases}
$$

From the problem (20) integrating the equation on a small interval centered on $x=a$ and using (31) we get the jump condition

$$
\left[\phi_{x}\right]_{a^{-}}^{a^{+}}-j l=d_{a} \sin (\phi(a, t))=d_{a} \sin \left(\frac{j l t}{\alpha d_{a}}\right) .
$$

On the other hand from (40) we get

$$
\begin{aligned}
{\left[\phi_{x}\right]_{a^{-}}^{a^{+}}-j l } & =a_{l} \sin \left(V_{l} t\right) \sin \left(V_{l} a\right)-a_{r} V_{r} \sin \left(V_{r} t\right) \sin \left(V_{r}(a-l)\right) \\
& \equiv C_{1} \sin \left(V_{l} t\right)-C_{2} \sin \left(V_{r} t\right)
\end{aligned}
$$

Since $C_{1}$ and $C_{2}$ are independent of $t$, this implies that $\sin \left(V_{l} t\right), \sin \left(V_{r} t\right)$ and $\sin \left(\frac{j l t}{\alpha d_{a}}\right)$ have the same period. We obtain,

$$
\frac{2 k_{l}+1}{2} \frac{\pi}{a}=\frac{j l}{\alpha d_{a}}=\frac{2 k_{r}+1}{2} \frac{\pi}{a-l}=V,
$$

if $a_{l}$ and $a_{r}$ are not equal to zero. Then one can write the solution as

$$
\phi(x, t)=\left\{\begin{array}{lc}
\phi_{v}(x, t)+a_{l} \sin (V t) \cos (V x) & x<a \\
\phi_{v}(x, t)+a_{r} \sin (V t) \cos (V(x-l)) & a<x .
\end{array}\right.
$$


where $\phi_{v}$ is the high voltage solution. Because of this the instantaneous voltage $\phi_{t}$ has a discontinuous first derivative at $x=a$.

Assuming that $\alpha=1$ we see that the consistency equation (41) implies

$$
(a-l) 2 k_{l}-2 a k_{r}=l
$$

There are very few solutions for $a$ rational et $l$ integer and for most $a$ there are none. Let us look at other possibilities. Notice first that space homogeneous functions $\left(a_{l}=0\right.$ or $\left.a_{r}=0\right)$ are solutions. Having said this it is easy to see that one can obtain left only or right only oscillations.

left only: $\phi(x, t)= \begin{cases}\phi_{v}(x, t)+a_{l} \sin (V t) \cos (V x) & x<a \\ \phi_{v}(x, t) & a<x\end{cases}$

right only: $\phi(x, t)= \begin{cases}\phi_{v}(x, t) & x<a \\ \phi_{v}(x, t)+a_{r} \sin (V t) \cos (V(x-l)) & a<x\end{cases}$

Again the instantaneous voltage at the junction has a discontinuous first derivative.

Fig. [3] shows the instantaneous voltage as a function of $x$ for different times for two such ohmic modes, the left only oscillation (left panel) and right and left oscillations (right panel). The left panel shows $\phi_{t}(x, t)$ for 60 values of time equidistributed between 0 and 10 . The parameters are the same as for Fig. 2 In this case we have $k_{l}=1$ so that about 1.5 periods exist to the left of $x=a$. Notice how the amplitude of $\phi_{t}$ is practically constant and equal to the average voltage $V=j l=1.37$ on the right of $x=a$. The right panel corresponds to a right and left oscillation for $a=2$ and we show three consecutives times, $t=0,0.125$ and 0.250 . Here $\frac{2 k_{l}+1}{2 k_{r}+1}=\frac{a}{l-a}=3 / 7$ so that we get about 1 period to the left of $x=a$ and two periods to the right of $x=a$. We show for comparison $V=\frac{\pi}{2}(=j l)$ the average voltage. Notice in both cases the discontinuity of $\phi_{t}$ at $x=a$.

Let us show that this is consistent with the whole equation (20), for the intensity $I=V / \alpha d_{a}$. Consider the left only solution

$$
\phi(x, t)= \begin{cases}V t+\frac{a_{l}}{V} \sin (V t) \cos (V x)-\frac{j}{2}\left[x^{2}-a^{2}\right] & x<a \\ V t-\frac{j}{2}\left[(x-l)^{2}+(a-l)^{2}\right] & x>a\end{cases}
$$

with, $V=\frac{2 k_{l}+1}{2} \frac{\pi}{a}$. On the interval $[0, a[$ (respectively $\left.] a, l]\right) \phi$ satisfies the linear wave equation $\phi_{t t}-\phi_{x x}=j$ with the boundary condition $\left.\phi_{x}\right|_{0}=0$ (respectively 
$\left.\left.\phi_{x}\right|_{l}=0\right)$. We now integrate (20) on $[0, l]$ to obtain

$$
\int_{0}^{a} \phi_{t t} d x+\int_{a}^{l} \phi_{t t} d x+\int_{0}^{l} \phi_{x x} d x+d_{a} \sin (V t)+\alpha d_{a} V=j l
$$

so that

$$
-a_{l} \sin (V t)[\sin (V x)]_{0}^{a}+0+0+d_{a} \sin (V t)=0 .
$$

The end result is

$$
(-1)^{k_{l}+1} a_{l} \sin (V t)=d_{a} \sin (V t)
$$

which implies $a_{l}=(-1)^{k_{l}+1} d_{a}$ and the equation is balanced.

\section{The junction mode}

The expression (35) a priori defined only for a given time interval can be $C^{\infty}$ extended for all times using the continuation

$$
f\left(t+k \frac{2 \alpha \pi}{\sqrt{\left(j / d_{a}\right)^{2}-1}}\right)=f(t)+k 2 \pi,
$$

where $k$ is an integer. The pseudo-period $T$ of $\phi_{a}$ is $T=\frac{2 \alpha \pi}{\sqrt{\left(j / d_{a}\right)^{2}-1}}$ so the voltage is $V=\frac{2 \pi}{T}=\frac{1}{\alpha} \sqrt{\left(j / d_{a}\right)^{2}-1}$. This can also be written

$$
j=\frac{d_{a}}{l} \sqrt{(\alpha V)^{2}+1}
$$

So we deduce from $A_{0}^{\prime \prime}(t)=0$ and the voltage expression (36) that $A_{0}(t)=$ $V t+C$.

Let us now calculate the contribution of the Fourier modes and give a solution to the whole equation (30). From (30) and the previous equality, we obtain:

$$
A_{p}^{\prime \prime}(t)+\left(\frac{p \pi}{l}\right)^{2} A_{p}(t)=-2 j c_{p}
$$

The solution of this equation, $A_{p}$ is:

$$
A_{p}(t)=\gamma_{p} \cos \left(\frac{p \pi}{l} t\right)+\beta_{p} \sin \left(\frac{p \pi}{l} t\right)-\frac{2 j l^{2}}{(p \pi)^{2}} c_{p}
$$

Now we have the expression of all the Fourier modes. We know that $\phi(a, t)=$ $\sum_{n=0}^{+\infty} A_{n}(t) c_{n}^{a}$. Substituting the Fourier modes (47) into this expression we obtain:

$$
\phi(a, t)=V t+C+\sum_{p=1}^{+\infty}\left[\gamma_{p} \cos \left(\frac{p \pi}{l} t\right)+\beta_{p} \sin \left(\frac{p \pi}{l} t\right)-\frac{2 j l^{2}}{(p \pi)^{2}} c_{p}\right] c_{p},
$$


The series in the last term is uniformly convergent so that the term can be collected in the form of a constant yielding the final simpler expression

$$
\phi(a, t)=V t+C_{2}+\sum_{p=1}^{+\infty}\left[\gamma_{p} c_{p} \cos \left(\frac{p \pi}{l} t\right)+\beta_{p} c_{p} \sin \left(\frac{p \pi}{l} t\right)\right]
$$

Introduce $\phi(a, t)-V t+C_{2}=u(t)$. We can choose $C_{2}$ as $\int_{0}^{T} u(t) d t=0$. Notice that $u$ is a $C^{\infty}$ and $T$-periodic function. We can make Fourier projection on it.

$$
u(t)=\sum_{p=1}^{+\infty}\left[\gamma_{p} c_{p} \cos \left(\frac{p \pi}{l} t\right)+\beta_{p} c_{p} \sin \left(\frac{p \pi}{l} t\right)\right]
$$

Until $c_{p} \neq 0$, we have

$$
\begin{aligned}
& \gamma_{p}=\frac{2}{c_{p} T} \int_{0}^{T} u(t) \cos \left(\frac{p \pi}{l} t\right) d t \\
& \beta_{p}=\frac{2}{c_{p} T} \int_{0}^{T} u(t) \sin \left(\frac{p \pi}{l} t\right) d t
\end{aligned}
$$

We can make the following remarks

1. $V=\frac{2 \pi}{T}=\frac{n \pi}{l}$ so $\frac{1}{T}=\frac{n}{2 l}$ and $u$ is $\frac{2 l}{n}$ periodic.

2. If $V=\frac{n \pi}{l}$ the terms of the Fourier series that aren't zero are the multiples of $n$.

3. If $c_{n}=0$ then the junction modes for $V=\frac{n \pi}{l}$ can't exist. For example when the junction is in the middle of the circuit, then $c_{n}^{a}=0$ for $n=2 k+1$ and junction modes exist only for $V=\frac{2 k \pi}{l}$ where $k$ is an integer (see left panel of figure 5).

4. When $\left|c_{n}\right|$ is very small, the circuit in the junction mode stores a lot of energy but is unstable. We will see this in the next section.

Now remember (28), we can give the explicit solution of the phase in the $n$th junction mode, up to a constant

$$
\phi(x, t)=\frac{n \pi}{l} t+\sum_{p=1}^{+\infty}\left[\gamma_{p} \cos \left(\frac{p n \pi}{l} t\right)+\beta_{p} \sin \left(\frac{p n \pi}{l} t\right)\right] \cos \left(\frac{p n \pi}{l} x\right) .
$$

Notice that the instantaneous voltage $\phi_{t}$ has a continuous derivative at the junction position $x=a$. Fig. 4 shows the instantaneous voltage $\phi_{t}(x, t)$ as a 
function of $x$ for 60 consecutive times for the same parameters as in Fig. 2 . Notice how the solution oscillates over the whole domain and that one cannot see the junction position. This type of motion is similar to the transparency observed in the reflection coefficient of a stripe [19] when an integer number of half-periods of the wave "fits" in the stripe.

\section{Single junction case: IV characteristics}

For a large enough time the system finds a stationary state where the energy provided by the input current is balanced by dissipation. This state is described by the IV characteristic curve. This is measured on real devices and it is therefore very important to understand its features.

To compute it we fix the current and run the system for about $t_{1}=3000$ time units so that all transients have died off. Then we compute the average voltage

$$
\left\langle\phi_{t}(a, t)\right\rangle_{t} \equiv \frac{\phi\left(a, t_{1}+T\right)-\phi\left(a, t_{1}\right)}{T}
$$

at the junction. The value $T$ is about 2000. The space average of the average

voltage in time $\left\langle\frac{1}{l} \int_{0}^{l} \phi_{t}(x, t) d x\right\rangle_{t}$ is compared to this quantity to ensure that the system is completely thermalized. Only then is the voltage recorded.

The IV curves are computed for 99 steps of increasing current $j$ from 0 to 4 starting with an initial condition $\phi(x, 0) \equiv 0$ and $\phi_{t}(x, 0) \equiv 0$. We then decrease the current from 4 to 0 . All plots show these two curves for increasing and decreasing current.

\subsection{Bounds for IV curves}

We have isolated two characteristic behaviors of the circuit. These allow us to bound the IV curves for a single junction in a microstrip. Fig. [5] shows two IV curves for a centered junction $a=5$ (left panel) and an off-centered junction $a=9$ in a microstrip of total length $l=10$. For each case we plot the limiting behaviors given by the ohmic mode and junction mode voltages (34) and (36). $V, j$ are bounded by the relations

$$
d_{a} \alpha V \leq j l \leq d_{a} \sqrt{(\alpha V)^{2}+1}, \text { or } \frac{1}{\alpha} \sqrt{\left(\frac{j l}{d_{a}}\right)^{2}-1} \leq V \leq \frac{j l}{d_{a} \alpha}
$$


The ohmic modes, (resp. the junction modes) only occur for voltages such that $V=V_{l}=\frac{2 k_{l}+1}{2} \frac{\pi}{a}$ or $V=V_{r}=\frac{2 k_{r}+1}{2} \frac{\pi}{a-l}$ (resp. $V=\frac{k \pi}{l}$ ). The voltage (horizontal) axis is labeled with the indices corresponding in each case to the junction modes. The ohmic modes are marked with ticks but not labelled. We always go from an ohmic mode to a junction mode and vice versa. As the current is increased the junction mode ceases to exist and the system then jumps to the closest ohmic mode. Notice the hysteresis obtained between the curve for increasing $j$ and the one for decreasing $j$. Both the junction voltage and ohmic mode voltages are fixed by the length of the system. On the other hand the current for which one obtains these voltages depends on the damping $\alpha$ and strength of the junction $d_{a}$. For fixed $d_{a}$, an decrease of $\alpha$ will tilt the IV curves towards the horizontal voltage axis and this will separate the cavity modes and cause large voltage jumps from a cavity mode to a far away ohmic mode and hysteresis. Conversely an increase of $\alpha$ will make the IV curves more vertical so that the voltage jumps and hysteresis will be reduced. Note also that as in the static case, a magnetic field has no effect on the IV curves for a single junction.

When the junction is centered as in the left panel of Fig. 5 the ohmic modes (resp. junction modes) correspond to the odd $V=(2 k+1) \pi / l$ (resp. even $V=2 k \pi / l)$ cavity modes so the voltage interval between a junction mode and its corresponding ohmic mode neighbors is constant. This does not happen when the junction is off-centered as in the right panel of Fig. 5 so that one can get very sharp resonances connecting an ohmic mode to its junction mode neighbor like the ones for $n=2,3$ and 4 . The resonance for $n=3$ was obtained by decreasing current. For larger values of $n$ the voltage separation becomes larger so that the resonances are softer.

\subsection{Study of resonances}

We have been able to bound the IV curves for our system. Now let us discuss the fine structure of the resonances, why some are sharp (see resonances 3,4 and 6 in Fig. 5) while others are not. To explain this one should consider the energy of the system and the Fourier modes. Multiplying (20) by $\phi_{t}$ and integrating from 0 to $l$ we obtain the work equation

$$
\partial_{t}\left[\frac{1}{2} \int_{0}^{l} \phi_{t}^{2}+\phi_{x}^{2} d x+d_{a}(1-\cos \phi(a, t))\right]=j \int_{0}^{l} \phi_{t} d x-d_{a} \alpha \phi_{t}^{2}(a, t)
$$


We can write (54) as $\partial_{t} E_{t} \equiv \partial_{t}\left(E_{p}+E_{j}\right)=P_{d c}+P_{q}$ where $E_{p}=\frac{1}{2} \int_{0}^{l}\left(\phi_{t}^{2}+\right.$ $\left.\phi_{x}^{2}\right) d x$ is the energy in the passive region, $E_{j}=d_{a}(1-\cos \phi(a, t))$ the Josephson energy, $P_{d c}=j \int_{0}^{l} \phi_{t} d x$ is the power given by the direct current and $P_{q}=$ $d_{a} \alpha \phi_{t}^{2}(a, t)$ is power dissipated by the quasiparticles. In figure [ we plot from top to bottom $E_{t}, E_{p}, P_{q}$ and $E_{j}$ for a centered junction $a=5$ (bottom panels) and an off-centered junction $a=9$ (top panels). The left panels show the junction mode $n=4$ and the right panels the corresponding ohmic mode for the same current. In the junction mode the quasi-particle term $P_{q}$ oscillates strongly and the Josephson energy $E_{j}$ is very anharmonic. In the ohmic mode $P_{q}$ is almost constant and $E_{j}$ is sinusoidal. In all cases the energy in the cavity $E_{p}$ is much larger than the junction energy $E_{j}$. When the junction is off-centered (top left panel) the energy stored in the cavity is much larger. This is due to the smallness of the coupling coefficient $c_{n}$ as we now show.

For that consider the phase $\phi(a, t)$ at the junction as given by (35). It is independent of the junction position $a$. Since we are in the junction mode $V=n \pi / l$ we write from (52)

$$
\phi(a, t)=\frac{n \pi}{l} t+\sum_{p=1}^{+\infty}\left[\gamma_{p} \cos \left(\frac{p n \pi}{l} t\right)+\beta_{p} \sin \left(\frac{p n \pi}{l} t\right)\right] c_{n p} .
$$

The first statement implies that the terms $\gamma_{p} c_{n p}$ and $\beta_{p} c_{n p}$ are constants independent of the junction position $a$ so that $\gamma_{p}$ and $\beta_{p}$ are proportional to $1 / c_{n p}$. As a consequence if $c_{n p}$ is small the junction mode of index $n$ corresponds to a large amplitude in the mode $A_{n p}$. The system accumulates this energy in the passive region and the resonance is very sharp.

Fig. 6] shows a plot as a function of time, in each panel, from top to bottom of the total energy $E_{t}$, the energy in the passive region $E_{p}$, the power dissipated by the quasi-particles $P_{q}$ and the Josephson energy $E_{j}$ for a centered junction $a=5$ (bottom panels) and an off-centered junction $a=9$ (top panels). The left panels show the junction mode $n=4$ and the right panels the corresponding ohmic mode for the same current. The coupling coefficient is small $c_{4}=0.3$ in the top panel while it is maximum $c_{4}=1$ in the bottom panel where the junction is centered. Therefore one expects a sharp resonance and a highly excited passive cavity for $a=9$ as opposed to $a=5$. This is indeed the case as one can see from the plot of $E_{p}$ in the left bottom and left top panels. The Josephson energy $E_{j}$ and the power dissipated by the junction are of the same 
order. In the ohmic regime this accumulation of energy in the cavity is absent. There is a small difference in $E_{p}$ between the two configurations because they have different lengths of passive regions. Note that the total energy is practically independent of time and so is the dissipation. There is an exact balance between the Josephson energy and the energy in the cavity $E_{p}$.

\section{The many junction case}

Assuming a general situation with magnetic field, a mixed current feed and many junctions in the micro-strip we can generalize our model to

$$
\phi_{t t}-\phi_{x x}+\sum_{j} d_{j} \delta\left(x-a_{j}\right)\left(\sin (\phi)+\alpha \phi_{t}\right)=\nu j
$$

with the boundary conditions $\left.\phi_{x}\right|_{x=0}=b-\frac{j l}{2}(1-\nu)$ and $\left.\phi_{x}\right|_{x=l}=b+\frac{j l}{2}(1-\nu)$. If $\nu=1$, In the model (55) $\nu=1$ (resp. $\nu=0$ ) corresponds to an overlap (resp. inline) current feed.

\subsection{Static case}

We have seen that for a single junction the maximum current $j l=d_{a}$ is reached for any value of the magnetic field. This is not true for two junctions (a SQUID)

where the maximum critical current if obtained for $b=0$ mod. $\frac{2 \pi n}{a_{2}-a_{1}} \quad$ where $n$ is an integer [22. Static solutions for many junctions in a 1D microstrip will be discussed in a forthcoming paper [23].

\subsection{High voltage case}

As in the case of a single junction, when the stationary state is reached we can assume $\phi_{t}=V$ and average out the $\phi_{t t}$ and $\sin (\phi)$ terms yielding the following boundary value problem for the time independent part $\phi_{v}$ of $\phi$

$$
\begin{array}{r}
-\phi_{v_{x x}}+\sum_{k=1}^{n} d_{k} \delta\left(x-a_{k}\right) \alpha V=\nu j, \\
\left.\phi_{v_{x}}\right|_{0, l}=b \mp \frac{j l}{2}(1-\nu),
\end{array}
$$

On each interval separating the junctions $\phi_{v_{x x}}=-\nu j$ so that $\phi_{v}$ is a second degree polynomial $\phi_{v}(x)=-(\nu j / 2) x^{2}+b x+d$. 
Integrating (56) from 0 to $l$ yields the voltage

$$
V=\frac{j l}{\alpha \sum_{k=1}^{n} d_{k}}
$$

Integrating (56) over small domains containing the junctions we obtain as usual the jump conditions in the derivatives

$$
\left[\phi_{v_{x}}\right]_{a_{i}^{-}}^{a_{i}^{+}}=\frac{d_{i j l}}{\sum_{k=1}^{n} d_{k}} .
$$

Using these remarks we can calculate $\phi_{v}$ for any number of junctions (for simplicity one can assume $\left.\phi_{v}\left(a_{1}\right)=0\right)$. Let's introduce $S(x)$, the spatial curvature of $\langle\phi\rangle_{t}$. For two junctions we get

$$
\phi_{v}(x)=\left\{\begin{array}{lr}
\frac{-\nu j}{2}\left(x^{2}-a_{1}^{2}\right)+\left(b-\frac{j l}{2}(1-\nu)\right)\left(x-a_{1}\right) & x \leq a_{1} \\
\frac{-\nu j}{2}\left(x^{2}-a_{1}^{2}\right)+\left(\frac{d_{1} j l}{d_{1}+d_{2}}+b-\frac{j l}{2}(1-\nu)\right)\left(x-a_{1}\right) & a_{1}<x<a_{2} \\
\frac{-\nu j}{2}\left(x^{2}-a_{2}^{2}\right)+\left(b-\frac{j l}{2}(1+\nu)\right)\left(x-a_{1}\right)+S_{2} & a_{2} \leq x
\end{array}\right.
$$

where $S_{2}=\frac{-\nu j}{2}\left(a_{2}^{2}-a_{1}^{2}\right)+\left(\frac{d_{1} j l}{d_{1}+d_{2}}+b-\frac{j l}{2}(1-\nu)\right)\left(a_{2}-a_{1}\right)$ is the phase difference between the second and first junction $\left(S_{2} \equiv \phi\left(a_{2}, t\right)-\phi\left(a_{1}, t\right)\right)$. The phase is then given by

$$
\phi(x, t)=\phi_{v}(x)+V t+C,
$$

manyj where $C$ is an integration constant. Notice that it is possible to find $\phi_{v}(x)$ for an arbitrary number of junctions and compute the phase difference $\phi_{v}\left(a_{i}\right)=S_{i} \equiv \phi\left(a_{i}, t\right)-\phi\left(a_{i-1}, t\right)$.

In Fig. 7 we show $\phi(x, t)$ for 6 consecutive values of time separated by $\delta t=0.1$ for a system of two junctions located respectively at $a_{1}=2$ and $a_{2}=6.2$ in a domain of length $l=10$ with current $j=1$ fed in overlap geometry $(\nu=1)$. One can see that the behavior is close to what is predicted by the above expression.

\section{3 $\quad$ Fourier representation}

We can use the high voltage solution to simplify (55). For that take $\psi=\phi+\phi_{v}$ so that (55) becomes

$$
\begin{aligned}
\psi_{t t}-\psi_{x x}+\sum_{k=1}^{n} d_{k} \delta\left(x-a_{k}\right)\left(\sin \left(\psi-S_{k}\right)+\alpha \psi_{t}-\frac{j l}{\sum_{k=1}^{n} d_{k}}\right) & =0 \\
\left.\psi_{x}\right|_{x=0 ; l} & =0 .
\end{aligned}
$$


The transformation using the high voltage solution concentrates the current on the junctions. In this representation inline or overlap configurations differ by the terms $S_{k}$.

Let us go back with Fourier series. Because of the homogeneous Neumann boundary conditions on [60), we can decompose $\psi$ on a cosine Fourier series

$$
\psi(x, t)=\sum_{n=0}^{+\infty} A_{n}(t) \cos \left(\frac{n \pi x}{l}\right)
$$

and obtain the following equations for the modes $A n$

$$
\begin{array}{r}
l A_{0}^{\prime \prime}+\sum_{j=1}^{n} d_{j}\left(\sin \left(\psi_{j}-S_{j}\right)+\alpha \psi_{j t}-\frac{j l}{\sum_{k=1}^{n} d_{k}}\right)=0, \\
A_{n}^{\prime \prime}+\left(\frac{n \pi}{l}\right)^{2} A_{n}+\frac{2}{l} \sum_{j=1}^{n} d_{j} c_{n}^{j}\left(\sin \left(\psi_{j}-S_{j}\right)+\alpha \psi_{j t}-\frac{j l}{\sum_{k=1}^{n} d_{k}}\right)=0,
\end{array}
$$

where $c_{n}^{j}=\cos \left(\frac{n \pi a_{j}}{l}\right), \quad \psi_{j} \equiv \psi\left(a_{j}, t\right), \quad \psi_{j t} \equiv \partial_{t} \psi\left(a_{j}, t\right)$.

\section{IV curves for two or more junctions}

\subsection{2 symmetrically placed junctions, limiting behaviors.}

In a symmetric circuit, we have $a_{2}=l-a_{1}$ and $d_{1}=d_{2}$. For simplicity we assume no magnetic field so that $b=0$ too. With this symmetry, it is easy to show that $c_{2 k}^{2}=c_{2 k}^{1}$ and $c_{2 k+1}^{2}=-c_{2 k+1}^{1}$. It is important to remark that when $b=0, S_{2}=0$. So there is no phase shift between the two junctions. Let us now go back to the Fourier modes, (62) becomes:

$$
d_{1}\left(\sin \left(\psi_{1}\right)+\alpha \psi_{1 t}+\sin \left(\psi_{2}\right)+\alpha \psi_{2 t}\right)-j l=-l A_{0}^{\prime \prime} .
$$

For $p=2 k$,

$$
\begin{aligned}
A_{2 k}^{\prime \prime}+\left(\frac{2 k \pi}{l}\right)^{2} A_{2 k} & =-\frac{2 c_{2 k}^{1}}{l}\left[d_{1}\left(\sin \left(\psi_{1}\right)+\alpha \psi_{1 t}+\sin \left(\psi_{2}\right)+\alpha \psi_{2 t}\right)-j l\right] \\
& =2 c_{2 k}^{1} A_{0}^{\prime \prime}
\end{aligned}
$$

We want to show that the following equation for $A_{2 k+1}$ is decoupled from the system of Fourier modes

$A_{2 k+1}^{\prime \prime}+\left(\frac{(2 k+1) \pi}{l}\right)^{2} A_{2 k+1}=\frac{-2 c_{2 k+1}^{1}}{l} d_{1}\left(\sin \left(\psi_{1}\right)-\sin \left(\psi_{2}\right)+\alpha\left(\psi_{1 t}-\psi_{2 t}\right)\right)$. 
For that we group together the even terms and odd terms of the Fourier series of $\psi_{1 t}$ and $\psi_{2 t}$ and define $s_{o}(t) \equiv \sum_{k=0}^{+\infty} c_{2 k}^{1} A_{2 k}(t)$ and $s_{e}(t) \equiv \sum_{k=0}^{+\infty} c_{2 k+1}^{1} A_{2 k+1}(t)$. Notice that:

$$
\psi\left(a_{1}, t\right)=s_{o}(t)+s_{e}(t) \text { and } \psi\left(a_{2}, t\right)=s_{o}(t)-s_{e}(t)
$$

Replacing $\psi_{1}$ and $\psi_{2}$ in equation (65), we obtain:

$$
A_{2 k+1}^{\prime \prime}+\left(\frac{(2 k+1) \pi}{l}\right)^{2} A_{2 k+1}=\frac{-4 c_{2 k+1}^{1}}{l} d_{1}\left(\cos \left(s_{e}\right) \sin \left(s_{o}\right)-\alpha s_{e}^{\prime}\right)
$$

This equations show that the system performs like a single centered junction with different coupling coefficients. So we can balance the Fourier modes in the junction mode: with initial conditions $\forall k, A_{2 k+1}(0)=0, A_{2 k+1}^{\prime}(0)=0$ we have $S_{2}(0)=0$ and as a consequence $A_{2 k+1}(t) \equiv 0, \forall t \in \Re^{+}$. This shows that the equations for the odd terms are decoupled from the system.

\section{Junctions modes.}

As we see, the system (62) behaves like a single junction: the odd terms are equal to zero and the even terms give the junction modes (see (35)):

$$
\phi_{1}=\phi_{2}=2 \arctan \left\{\frac{d_{1}}{j l}\left[\tan \left(\frac{1}{2 \alpha} V_{j}\left(t+C_{2}\right)\right) V_{j}+1\right]\right\},
$$

with $V_{j}=\sqrt{\left(\frac{j l}{d_{1}}\right)^{2}-1}=\frac{n \pi}{l}$.

Notice that the two junctions are perfectly synchronized. In a non-symmetric situation, junctions modes for both junctions are particular cases.

\section{Ohmic modes.}

Choosing $S_{2}=0$ allow us to show again analytical solutions. Assuming $b=0$, there are three types of solution, given by different oscillations of the phase:

1. The phase oscillates between the junctions so that $V=\frac{(2 k+1) \pi}{a_{2}-a_{1}}$. Due to the symmetry of the problem, we cannot find solutions with $V=\frac{2 k \pi}{a_{2}-a_{1}}$.

2. The phase oscillates outside the junctions: $V=\frac{(2 p+1) \pi}{2 a_{1}}$.

3. The phase oscillates in all the passive parts so that $V=\frac{(2 k+1) \pi}{a_{2}-a_{1}}=\frac{(2 p+1) \pi}{2 a_{1}}$. This is only possible if $a_{2}-a_{1}$ is a fraction of $l-a_{2}$.

To conclude in the symmetric case, we know limit IV curves with exact solutions. See the left panel of Fig [1]. In the general case, we cannot find exact solutions, we have near ohmic mode solutions and peaks at the expected resonant voltages $V=\frac{n \pi}{l}$. 


\subsection{The non-symmetric cases: IV curves}

The IV curve obtained for a circuit of two junctions placed in a non symmetric fashion confirms the existence of ohmic modes at the expected voltages. It is still possible to characterize the IV curves using ohmic modes and junction modes even if there are only approximate. Without symmetry and when $S_{2}=0[\pi]$ we can obtain exactly any one of ohmic modes discussed in previous section. In addition to the symmetric case there two new possibilities where $V$ can be computed:

1. The phase oscillates between the junctions and to the right of $a_{1}$. See the left panel of Fig 8 as an example.

2. The phase oscillates to the left of $a_{2}$ and between the junctions.

In Fig 8 the middle panel shows the second point of the symmetric case and the right panel illustrates the case where the phase oscillates between the junctions for the non-symmetric case.

Peaks appear at the junction modes $V=\frac{k \pi}{l}$, but they are in general smaller than in the symmetric cases. Fig 9 shows the IV curves for two non symmetrically placed junctions and indeed one can see that the height of the resonances is lower than one for two decoupled junctions (the upper curve in the three panels). By varying the junction critical current (the coefficients $d_{1}$ and $d_{2}$ ) we show that the resonances are linked to one of the two junctions. To understand

this phenomenon, go back to the coupling coefficients $c_{n}^{j}$. For the second resonance in Fig. 9 $\left|c_{2}^{1}\right|=1,\left|c_{2}^{2}\right|=0.73$ so that the junctions are strongly coupled to the system. This is like in the symmetric case but it is an exception. The IV curve shows that they are together in junction mode (or very close). Consider now the third resonance. The coupling coefficients are $c_{3}^{1}=0$ and $c_{3}^{2}=0.90$ so that the junction $a_{1}$ is decoupled from the system, we can assume ohmic behavior for it and $a_{2}$ is in junction mode. The fourth resonance corresponds to the reverse situation, $c_{4}^{1}=1, c_{4}^{2}=0.06$ where $a_{2}$ is decoupled.

To confirm this, we plot in Fig. 10] $\phi_{1}$ and $\phi_{2}$ for the resonances 3 and 4 with $d_{1}=0.7, d_{2}=0.3$ (left panel of Fig. 9) and $d_{1}=d_{2}=0.5$ (middle panel of Fig. 9). Together with the numerical results we plot the junction mode solution (35) in dashed line. For the top panel (third resonance) one can see that the junction $a_{2}$ follows closely this behavior as opposed to $a_{1}$ which is in ohmic mode. The 
situation is reversed for the fourth resonance as shown in the bottom panels.

For resonances 2, 3 and 4 , the value of the critical current $d_{i}, i=1,2$ does not modify appreciably the behavior of the phase at the junctions (remark that $S_{2} \neq 0$ but stay small).

For a general resonance the junctions will work in different modes, one will be closer to a junction mode while the other will be closer to an ohmic mode. We call this mode a resistor-junction mode.

\section{Resistor-junction modes.}

Resistor-junction modes exist if the circuit has one junction heavily coupled while the other is decoupled. In this case, the system behaves almost like a single junction circuit. This is why these modes are associed to voltages $V=\frac{k \pi}{l}$.

If the junction $a_{i}$ is in ohmic mode, then $\left[\phi_{x}\right]_{a_{i}^{-}}^{a_{i}^{+}}=d_{k} \sin \left(V t+C_{1}\right)+C_{k}$. So that we can only have only one harmonic in the circuit. Whereas a junction mode exists only with an infinity of harmonics. So the system reaches a constructive equilibrium. For example in all the panels of Fig. 10 we plot the exact junction mode to compare. The observation of the phases in the junction mode indicates that it lingers more around $(2 k+1) \pi / 2$ than the expression (35). This gives for the IV curves of Fig. 9 resonances that are slightly higher than expected. This summarizes the influence of one junction on the other.

\section{$8.3 \pi$-junctions and magnetic field effects}

We now consider the situation where the critical current $d_{i}$ for some $i$ can be negative. Then the ith junction is a so-called $\pi$ junction for which the Josephson relation is given by $\sin (\phi+\pi)$ instead of $\sin (\phi)$. In this case we will show that the dynamics is very different than the one where all the $d_{i}$ are positive. Therefore combining $\pi$ and standard junctions provides a way to change the resonant frequencies of the array. Another important parameter which we have not varied up to now is the magnetic field $b$. We will see that a change of $b$ changes the IV characteristic in a way that is similar to changing the critical current density of some of the junctions in the array. This is another way to tune the array to resonate on specific frequencies.

We now illustrate these specific points on some examples. Consider an array 
of $N$ point junctions described by

$$
\phi_{t t}-\phi_{x x}+\sum_{j=1}^{N} d_{j} \delta\left(x-a_{j}\right)\left(\sin (\phi)+\alpha \phi_{t}+c \phi_{t t}\right)=\nu j,
$$

with the boundary conditions $\left.\phi_{x}\right|_{x=0}=b+\frac{j l}{2}(1-\nu)$ and $\left.\phi_{x}\right|_{x=l}=b-\frac{j l}{2}(1-\nu)$. First note that if $d_{i}<0$ for all i's then we can take $\psi=\phi+\pi$ to get us back to the standard case $d_{i}>0$. We therefore obtain the same IV curve as when all the $d_{i}>0$.

An interesting case corresponds to $N=2$. Let us consider the effect of an additional magnetic field $b^{\prime}$ on the IV curve. For that introduce $\psi(x, t)=$ $\phi(x, t)+f(x)$ where $f(x)=b^{\prime}\left(x-a_{1}\right)$. The equation verified by $\psi$ is

$\psi_{t t}-\psi_{x x}+d_{1} \delta\left(x-a_{1}\right)\left(\sin \left(\psi+f_{1}\right)+\alpha \psi_{t}\right)+d_{2} \delta\left(x-a_{2}\right)\left(\sin \left(\psi+f_{2}\right)+\alpha \psi_{t}\right)=\nu j$,

with the boundary conditions $\left.\psi_{x}\right|_{x=0}=b+b^{\prime}+\frac{j l}{2}(1-\nu)$ and $\left.\psi_{x}\right|_{x=l}=b+b^{\prime}-$ $\frac{j l}{2}(1-\nu)$. When $f_{1}=0$ and $f_{2}=2 \pi$ ie when $b^{\prime}=2 \pi /\left(a_{2}-a_{1}\right)$ we obtain the same equation for $\phi$ and $\psi$ apart from the boundary conditions. The IV curves for the two magnetic fields $b=0$ and $b=2 n \pi /\left(a_{2}-a_{1}\right)$ where $n$ is an integer are then identical. Now if one chooses a magnetic field $b=\pi /\left(a_{2}-a_{1}\right)$ then the phases are shifted by $\pi$ one from the other and this is like changing the sign of one of the $d_{i}$. In practice these results only makes sense for magnetic fields for which the size of the junctions can be neglected. This is the limit of our approach.

Fig. 11]shows two IV curves for two symmetrically placed junctions $a_{1}=2$ et $a_{2}=8$ such that $b=0 d_{1}=d_{2}=0.5$ corresponding to standard junctions (left panel) and $b=0 d_{1}=-d_{2}=0.5$ corresponding to a standard junction and a $\pi$ junction (right panel). We observe in the left panel resonances (junction modes) every $\frac{2 k \pi}{l}$ exactly as for a centered junction. In the right panel, resonances (junction modes) are found every $\frac{(2 k+1) \pi}{l}$. The $\pi$ phase shift between the two junctions implies that odd harmonics are highly coupled and even harmonics are decoupled from the system. We could have obtained the same change in the nature of the resonances by using the standard array (left panel) and changing the magnetic field to $b=\pi /\left(a_{2}-a_{1}\right)$. As the field is changed one then goes continuously from an IV curve with only even resonances to an IV curve with only odd resonances. 
Now let us consider how these results obtained for $N=2$ affect the behavior of an array of $N>3$ junctions. If there is a $d$ such that $a_{i+1}-a_{i}=n_{i} d$ where $n_{i}$ is an integer for all $i$ then the IV curves for $b=0$ will be the same as for $b=2 \pi / d$. If one chooses $b=\pi / d$ the junctions such that $f_{i}=\pi \bmod .2 \pi$ will be reversed while the ones for which $f_{i}=2 \pi$ mod. $2 \pi$ will not be affected. This is similar to a flute which is tuned by changing nodes.

\section{Conclusion}

We have introduced a simple and general model using a wave equation with delta distributed sine nonlinearities to describe the dynamics of point Josephson junctions in a 1D microstrip. This can be extended to $\pi$ junctions for which the current density can be either positive or negative in the domain. We can also apply it to situations where the current density in each junction is different.

For a single junction we have shown two limiting behaviors, the ohmic mode where the junction behaves as a resistor driven by the cavity, separating waves and the junction mode where the Josephson element is driving the cavity. These limiting behaviors have allowed us to bound the IV curves and understand the observed resonances. When another junction is present in the cavity, this simple classification is changed in general and we observe ohmic modes and combined junction/ohmic modes. The existence or not of the nth resonance is connected to the value of the Fourier coefficient $c_{n}^{a}=\cos n \pi a / l$.

These results carry over to the case of an array with many junctions where it is possible to choose a voltage where one of the junctions is inactive and another is in junction mode. We also use the analysis to understand the effect of an external magnetic field and the influence of having 0 or $\pi$ junctions. We believe that such a device composed of normal and $\pi$ junctions placed at specific locations in a microstrip can be used to great advantage for specific applications like resonators and frequency mixers.

\section{References}

[1] B. D. Josephson, Phys. Lett. 1, 251, (1962). 
[2] K. Likharev, Dynamics of Josephson junctions and circuits, Gordon and Breach, (1986).

[3] A. Barone and G. Paterno, Physics and Applications of the Josephson effect, J. Wiley, (1982).

[4] M. H. Chung and M. Salez, in Proc. 4th European Conference on applied superconductivity, EUCAS 99, 651, (1999).

[5] A. Benabdallah, J. G. Caputo and N. Flytzanis, Physica D 161, 79-101, (2002)

[6] A. Benabdallah and J. G. Caputo, J. of Applied Physics, 92, nb. 7, (2002).

[7] T. Lindstrom et al, Phys. Rev. Lett. 90, 117021, (2003).

[8] N. Lazarides, Phys. Rev. B 69, 212501, (2004).

[9] E. Goldobin et al, Phys. Rev. Lett. 92, 570051, (2004).

[10] G. Rotoli, Phys. Rev. B 68, 052505, (2003).

[11] H. Susanto and S. A. van Gils, Phys. Rev. B 69, 92507, (2004).

[12] E. Almaas and D. Stroud, Phys. Rev. B, 63, 144522, , (2001).

[13] G. Filatrella and N. F. Pedersen, Physica C, 372-376, 11, (2002).

[14] S. P. Benz and C. J. Burroughs, Appl. Phys. Lett. 58, (1991).

[15] P. Barbara, A. B. Cawthorne, S. V. Shitov and C. J. Lobb, Phys. Rev. Lett. 82, 1963, (1999).

[16] S. V. Vasilic, P. Barbara, S. V. Shitov and C. J. Lobb, Phys. Rev. B 65, 180503, (2002).

[17] E. Hairer, S. P. Norsett and G. Wanner. Solving ordinary differential equations I (Springer-Verlag, 1987).

[18] H. S. Carslaw, An introduction to the theory of Fourier series and integrals , 3rd revised edition, Dover (1950).

[19] H. Lamb, Elements of soliton theory, Wiley, (1983). 
[20] L. Maurice, Statique de jonctions Josephson dans un microstrip, Memoire de DEA, Insa de Rouen, (2003).

[21] M.F. Boussaha, Développement d'un démonstrateur de récepteur hétérodyne submillimétrique ultra-large bande à base de jonctions Supraconducteur-Isolant-Supraconducteur (SIS), refroidi à 4,2K., $\mathrm{PhD}$ thesis, Université de Paris 6, (2003).

[22] J. G. Caputo and Y. Gaididei, Physica C, Two point Josephson junctions in a superconducting stripline: static case., Physica C 402, 160-173, (2004).

[23] J. G. Caputo and L. Loukitch, in preparation.

\section{Appendix}

\subsection{Continuum limit.}

We integrate (16) from $a-h$ to $a+h$

The first term yields

$$
\begin{aligned}
\int_{a-h}^{a+h} \phi_{t t} d x & =\int_{a-h}^{a+h} \phi_{t t}(a)+(x-a) \phi_{t t x}(a)+O\left(|x-a|^{2}\right) d x \\
& =2 h \phi_{t t}(a)+4 h^{2} \phi_{t t x}(a)+O\left(h^{3}\right)
\end{aligned}
$$

The second one

$$
\int_{a-h}^{a+h} \phi_{x x} d x=\phi_{x}(a+h)-\phi_{x}(a-h)
$$

and the third one

$$
\begin{aligned}
\int_{a-h}^{a+h}\left[\sin (\phi)+\alpha \phi_{t}+c \phi_{t t}\right] d x= & \int_{a-h}^{a+h} \sin \left(\phi(a)+(x-a) \phi_{x}(a)+O_{1}\left(|x-a|^{2}\right)\right) \\
& +\alpha \phi_{t}(a)+\alpha(x-a) \phi_{x t}(a)+O_{2}\left(|x-a|^{2}\right) \\
& +c \phi_{t t}(a)+c(x-a) \phi_{x t t}(a)+O_{3}\left(|x-a|^{2}\right) d x \\
= & \int_{a-h}^{a+h} \sin \left(\phi(a)+(x-a) \phi_{x}(a)+O_{1}\left(|x-a|^{2}\right)\right) d x \\
& +\alpha \phi_{t}(a)+\alpha h \phi_{x t}(a)+c \phi_{t t}(a)+\operatorname{ch} \phi_{x t t}(a)+O_{4}\left(h^{2}\right)
\end{aligned}
$$


We now expand the sine term

$$
\begin{aligned}
\sin \left(\phi(a)+(x-a) \phi_{x}(a)\right)= & \sin (\phi(a)) \cos \left((x-a) \phi_{x}(a)\right) \\
& +\sin \left((x-a) \phi_{x}(a)\right) \cos (\phi(a))
\end{aligned}
$$

so that

$$
\begin{aligned}
& \int_{a-h}^{a+h} \sin \left(\phi(a)+(x-a) \phi_{x}(a)\right) \\
= & \int_{a-h}^{a+h} \sin (\phi(a)) \cos \left((x-a) \phi_{x}(a)\right)+\sin \left((x-a) \phi_{x}(a)\right) \cos (\phi(a)) d x \\
= & \sin (\phi(a)) \frac{2}{\phi_{x}(a)} \sin \left(h \phi_{x}(a)\right)-0
\end{aligned}
$$

Consider the limit of this term when $h \rightarrow 0$. We have:

$$
d_{a} \frac{1}{2 h} \frac{2}{\phi_{x}(a)} \sin \left(h \phi_{x}(a)\right) \rightarrow d_{a},
$$

so that the equation for the point junction is

$$
\phi_{x}(a+h)-\phi_{x}(a-h)+d_{a}\left(\sin (\phi(a))+\alpha \phi_{t}(a)+c \phi_{t t}(a)\right)=j,
$$

which is consistent with the model (19).

$$
\phi_{t t}-\phi_{x x}+d_{a} \delta(x-a)\left(\sin (\phi)+\alpha \phi_{t}+c \phi_{t t}\right)=j
$$

\subsection{Method of lines}

The basis of the method is to discretize the spatial part of the operator and keep the temporal part as such. We thereby transform the partial differential equation into a system of ordinary differential equations. This method allows to increase the precision of the approximation in time and space independently and easily. In our case the operator is a distribution so that the natural way to give it meaning is to integrate it over a volume. We therefore choose as space discretisation the finite volume approximation where the operator is integrated over reference volumes. The value of the function is assumed constant in each volume.

As solver for the system of differential equations, we use the Runge-Kutta method of order 4-5 introduced by Dormand and Prince implemented as the Fortran code DOPRI5 by Hairer and Norsett [17] which enables to control the local error by varying the time-step. 
We first transform (20) into a system of first order partial differential equations We write $\psi(x, t)=\phi_{t}(x, t)$.

$$
\left\{\begin{aligned}
\psi(x, t) & =\phi_{t}(x, t) \\
\psi_{t}(x, t) & =\phi_{x x}(x, t)-\delta(x-a)\left(d_{a} \sin (\phi(x, t))+\alpha \psi(x, t)\right)+j
\end{aligned}\right.
$$

with the boundary conditions : $\left.\phi_{x}\right|_{\frac{l}{2}}=\left.\phi_{x}\right|_{-\frac{l}{2}}=0$.

For simplicity we will describe the implementation of the finite volume discretisation in the case of a single junction. We introduce reference volumes $V_{k}$ whose centers we call $x_{k}, 1 \leq k \leq n n$. The discretisation points are placed such that the point $x_{n g+1}$ is at the junction, $\left(x_{n g+1}=a\right)$. We thus define $x_{k}$ and $V_{k}$ using the following identities

$$
\left.V_{k}=\right] x_{k}-\frac{h_{g}}{2}, x_{k}+\frac{h_{g}}{2}[, \quad 0<k<n g+1
$$

with $(n g+1) h_{g}=a$

$$
\left.V_{k}=\right] x_{k}-\frac{h_{d}}{2}, x_{k}+\frac{h_{d}}{2}[, \quad n g+1<k<n n+1
$$

with $(n n-n g) h_{d}=l-a$. Finally at the junction, $k=n g+1$

$$
\left.V_{k_{n g+1}}=\right] x_{n g+1}-\frac{h_{g}}{2}, x_{n g+1}+\frac{h_{d}}{2}[.
$$

$n n, n g$ and $n d$ are respectively the total number of discretisation points, the number of points to the left of the junction and the number of points to the right.

For a fixed $\mathrm{t}$, we assume $\phi(x, t)$ to be constant on each volume $V_{k}$, so that

$$
\int_{x_{k}-\frac{h}{2}}^{x_{k}+\frac{h}{2}} \phi(x, t) d x=h \phi\left(x_{k}, t\right), \quad \text { with } h=h g \text { or } h=h d
$$

Integrating over $V_{k}$ yields:

1. In the linear part of the PDE $: 0<k<n n+1$ and $k \neq n g+1$ :

$$
\left\{\begin{aligned}
\psi\left(x_{k}, t\right) & =\phi_{t}\left(x_{k}, t\right) \\
\psi_{t}\left(x_{k}, t\right) & =\frac{\phi\left(x_{k+1}, t\right)-2 \phi\left(x_{k}, t\right)+\phi\left(x_{k-1}, t\right)}{h^{2}}+j
\end{aligned}\right.
$$

with $h=h g$ for $0<k<n g+1$ or $h=h d$ for $k>n g+1$. We recognize the usual discretisation of the second derivative. 


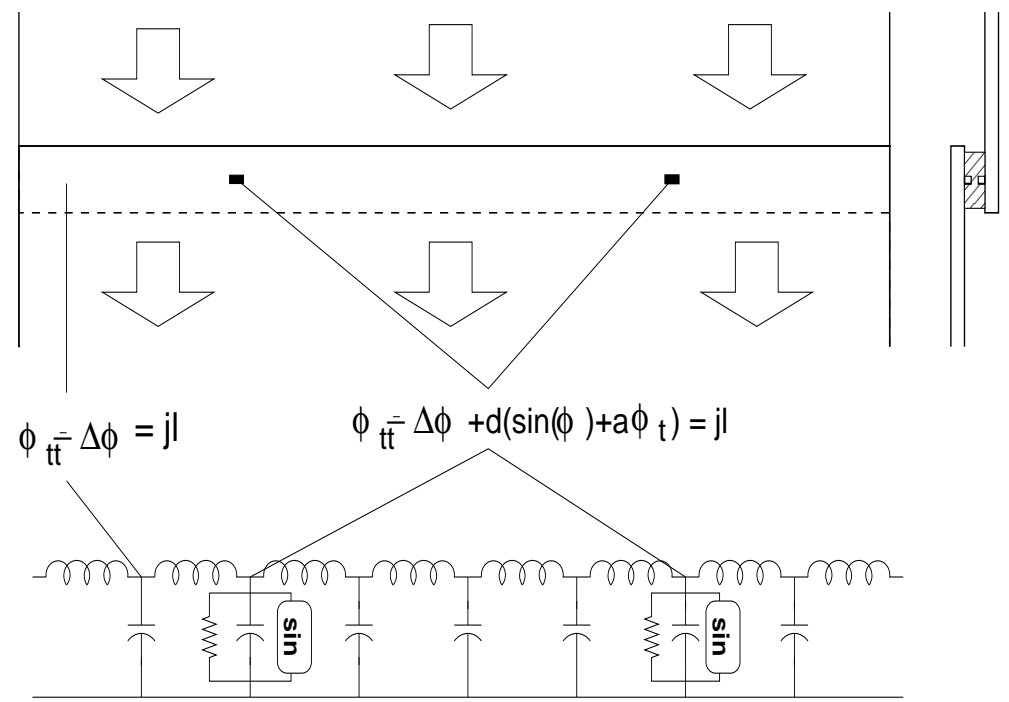

Figure 1: Left top pannel, a schematic drawing of a narrow 2D microstrip line containing two small Josephson junctions, right top panel section at one of the junction. The bottom panel presents the equivalent 1D electric circuit.

2. At the junction: $k=n g+1$, we obtain

$$
\begin{array}{r}
\int_{x_{n g+1}-\frac{h_{g}}{2}}^{x_{n g+1}+\frac{h_{d}}{2}} \delta(x-a)\left(d_{a} \sin (\phi(x, t))+\alpha \phi_{t}(x, t)\right)= \\
d_{a} \sin \left(\phi\left(x_{n g+1}, t\right)\right)+\alpha \phi_{t}\left(x_{n g+1}, t\right)
\end{array}
$$

So that the final system is:

$$
\left\{\begin{aligned}
\psi\left(x_{n g+1}, t\right)= & \phi_{t}\left(x_{n g+1}, t\right) \\
\psi_{t}\left(x_{n g+1}, t\right)= & \frac{4}{h g+h d}\left(\frac{\phi\left(x_{n g+2}, t\right)-\phi\left(x_{n g+1}, t\right)}{h g / 2}-\frac{\phi\left(x_{n g+1}, t\right)-\phi\left(x_{n g}, t\right)}{h d / 2}\right) \\
& -\frac{2}{h g+h d}\left(d_{a} \sin \left(\phi\left(x_{n g+1}, t\right)\right)+\alpha \phi_{t}\left(x_{n g+1}, t\right)\right)+j
\end{aligned}\right.
$$

The ODE system [68] [69) is then integrated numerically using the DOPRI5 integrator. 

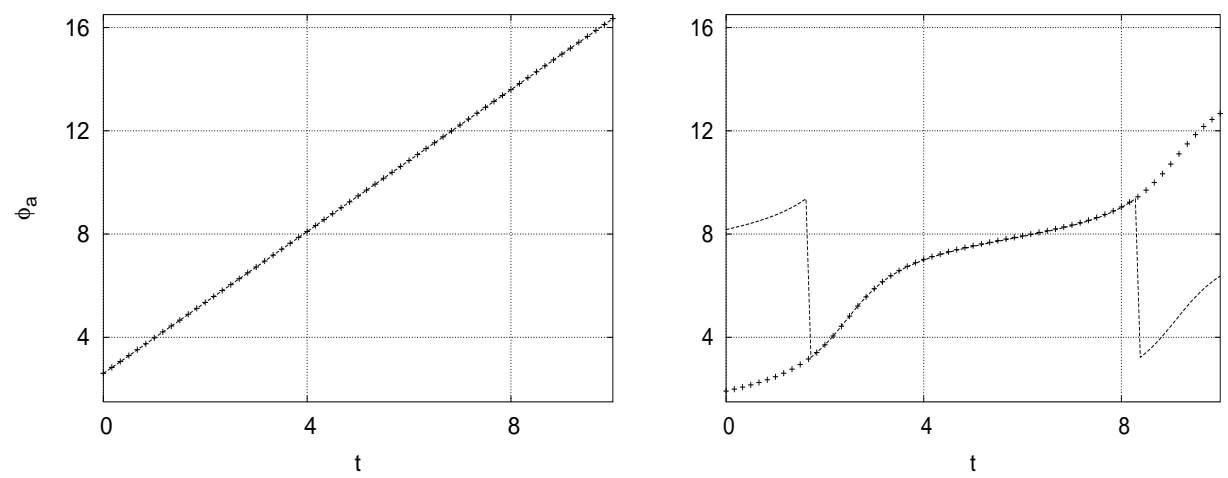

Figure 2: plot of $\phi_{a}(t)$ for $j=0.137414$ for a junction position $a=3.43, d_{a}=1$ and length $l=10$. The left panel shows the ohmic mode regime and the right panel the junction mode regime. The numerical solution of (20) is shown with the crosses while the analytical estimates (33) and (35) are in dashed line.
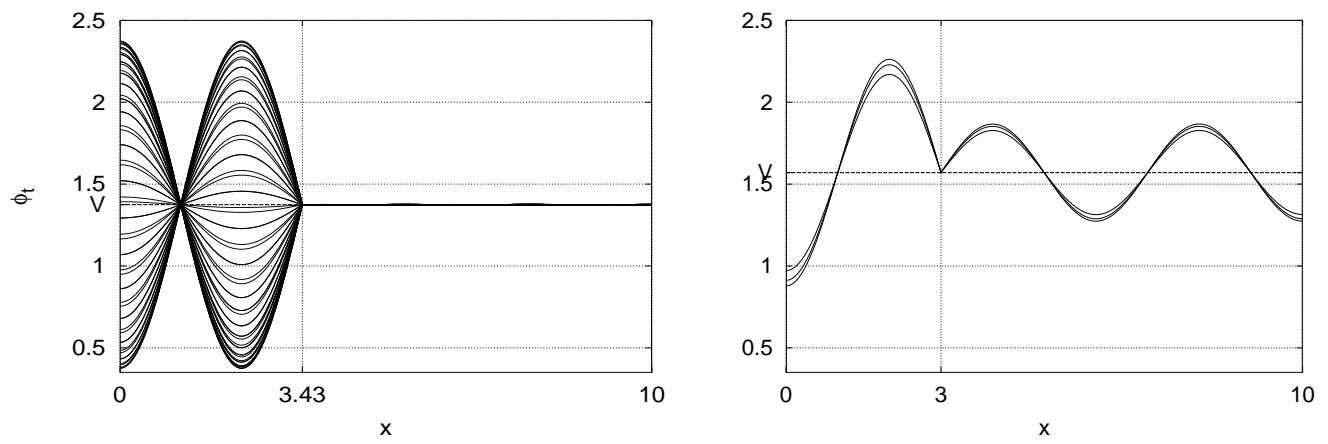

Figure 3: Instantaneous voltage $\phi_{t}(x, t)$ as a function of $x$ for different times for the ohmic mode: left only oscillation for the junction shown in Fig. 2 placed at $a=3.43$ (left panel) and left and right oscillation for a junction placed at $a=2$ (right panel). The average voltage is indicated in both figures, $V=j l=1.37$ in the left panel (same as in Fig. 22) and $V=\frac{\pi}{2}(=j l)$ on the right panel. The other parameters are $\alpha=1$, and $d_{a}=1$. 


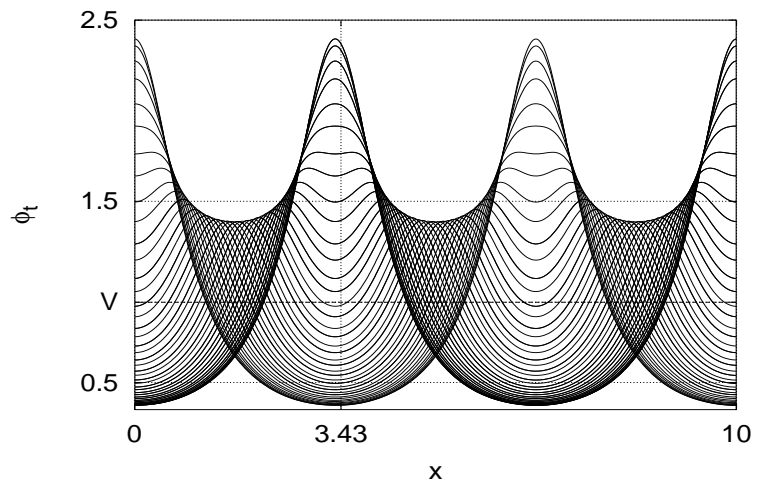

Figure 4: Instantaneous voltage $\phi_{t}(x, t)$ in the junction mode as a function of $x$ for 60 values of time equidistributed between 0 and 10. The parameters are the same as for Fig. 1. The average voltage $V$ is indicated on the vertical axis and the junction's position is indicated on horizontal axis.
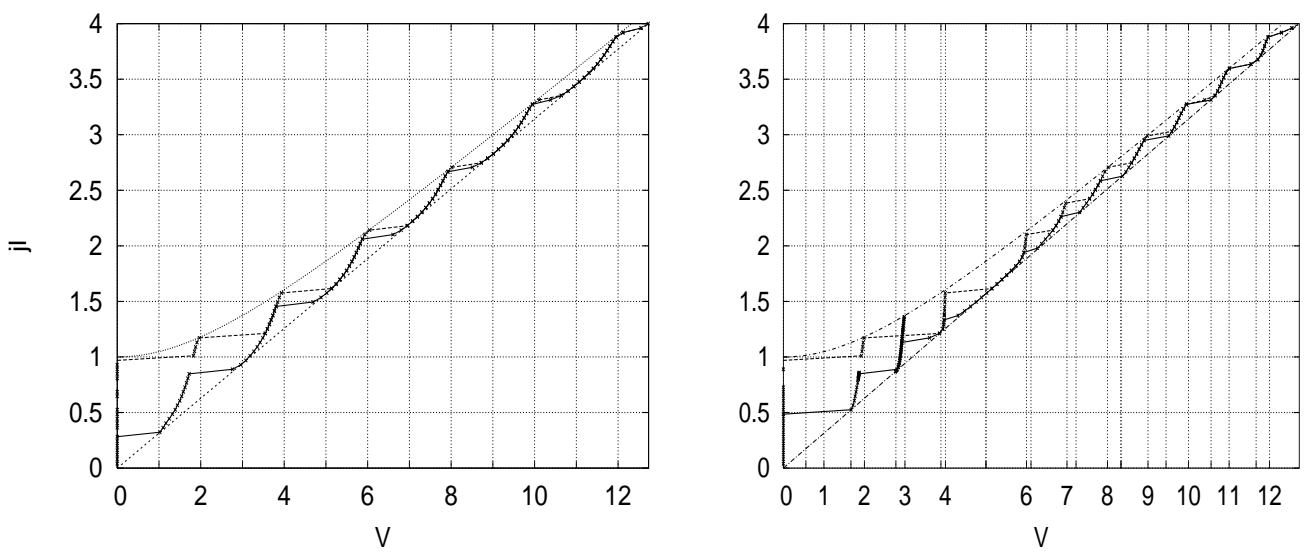

Figure 5: Two IV characteristic for a single junction placed in the center of the microstrip at $a=5$ (left panel) and near the right edge at $a=9$ (right panel). Only the positions of the junction modes are indicated in units of $\frac{\pi}{10}$. The other ticks correspond to the ohmic modes. For each panel two curves are presented, the top one is for increasing current while the bottom one is for a decreasing current showing a clear hysteresis. The minimum value of the current is 0 , the maximum is 4 and the stepping is $\frac{4}{99}$. The other parameters are $d_{a}=\alpha=1$. 

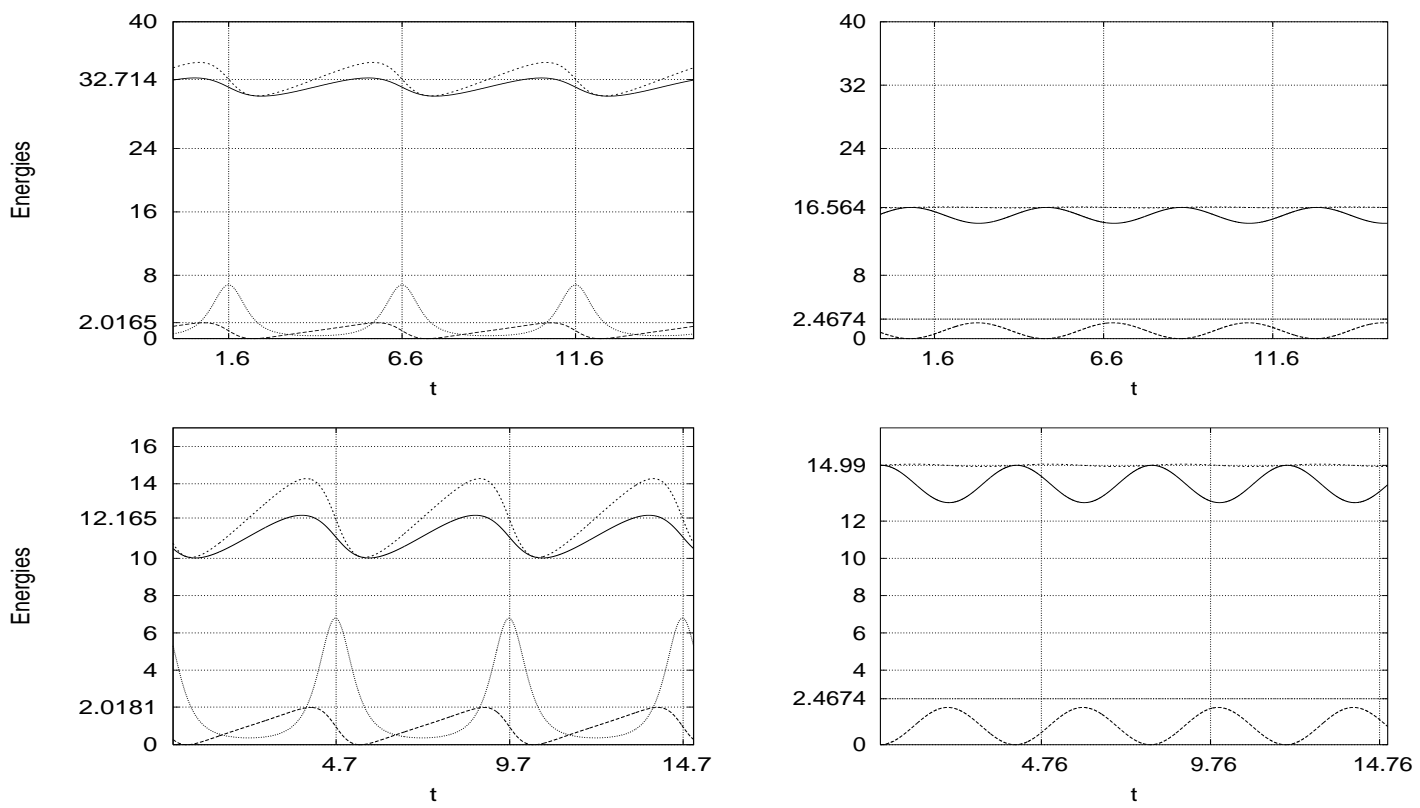

Figure 6: Plot as a function of time, in each panel, from top to bottom of the total energy $E_{t}$, the energy in the passive region $E_{p}$, the power dissipated by the quasi-particles $P_{q}$ and the Josephson energy $E_{j}$ for a centered junction $a=5$ (bottom panels) and an off-centered junction $a=9$ (top panels). The left panels show the junction mode $n=4$ and the right panels the corresponding ohmic mode for the same current. The averages of $E_{t}$ and $E_{j}$ are shown on the $y$ axis.

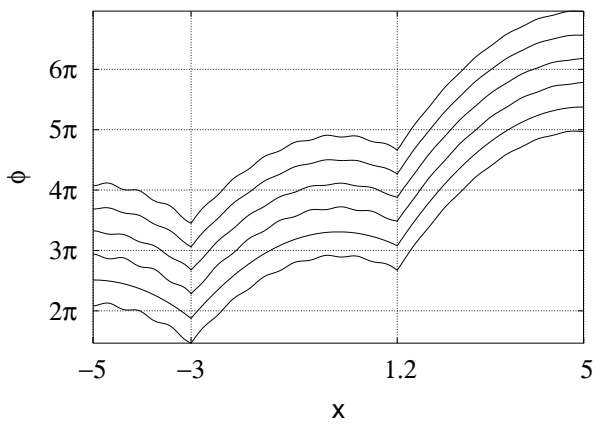

Figure 7: High voltage case: plot of $\phi(x, t)$ as a function of $x$ pour 6 consecutive times separated by $\Delta t=0.1$. The two junctions are located at $a_{1}=2$ and $a_{2}=6.2$. The other parameters are $l=10, j=1$, and $\alpha=0.5$. 

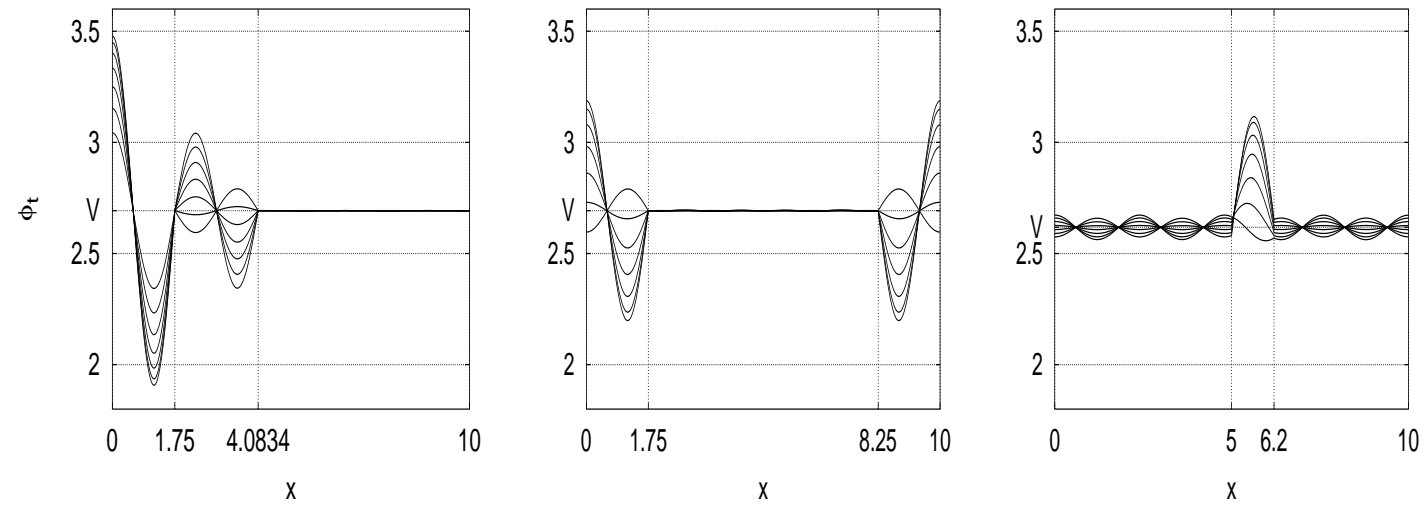

Figure 8: Three types of ohmic modes obtained for two junctions in a microstrip.

The positions of the junctions are given in the pictures. We plot $\phi_{t}(x, t) \forall x \in$ $[0, l]$ for six consecutive times. The parameters of equation (20) are $\lambda=0.5$ and $\alpha=1$. All figures have been obtained with the same current $j l=2.6927937$.
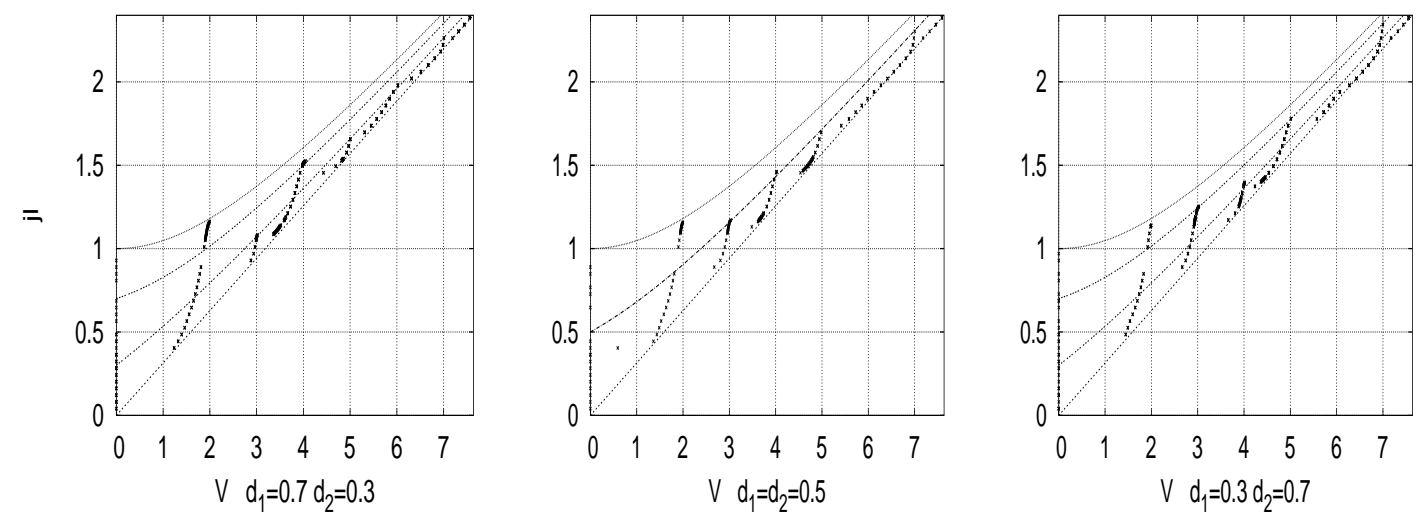

Figure 9: IV characteristic curves for two junctions placed in $a_{1}=5$ and $a_{2}=$ 6.2. The critical current densities are $d_{1}=0.7, d_{2}=0.3$ for the left panel, $0.5,0.5$ for the middle panel and $0.3,0.7$ for the right panel. We plot on all figures $j l=\sqrt{V^{2}+1}, j l=V, j l=d_{1} \sqrt{V^{2}+1}+d_{2} V$ and $j l=d_{2} \sqrt{V^{2}+1}+d_{1} V$. 

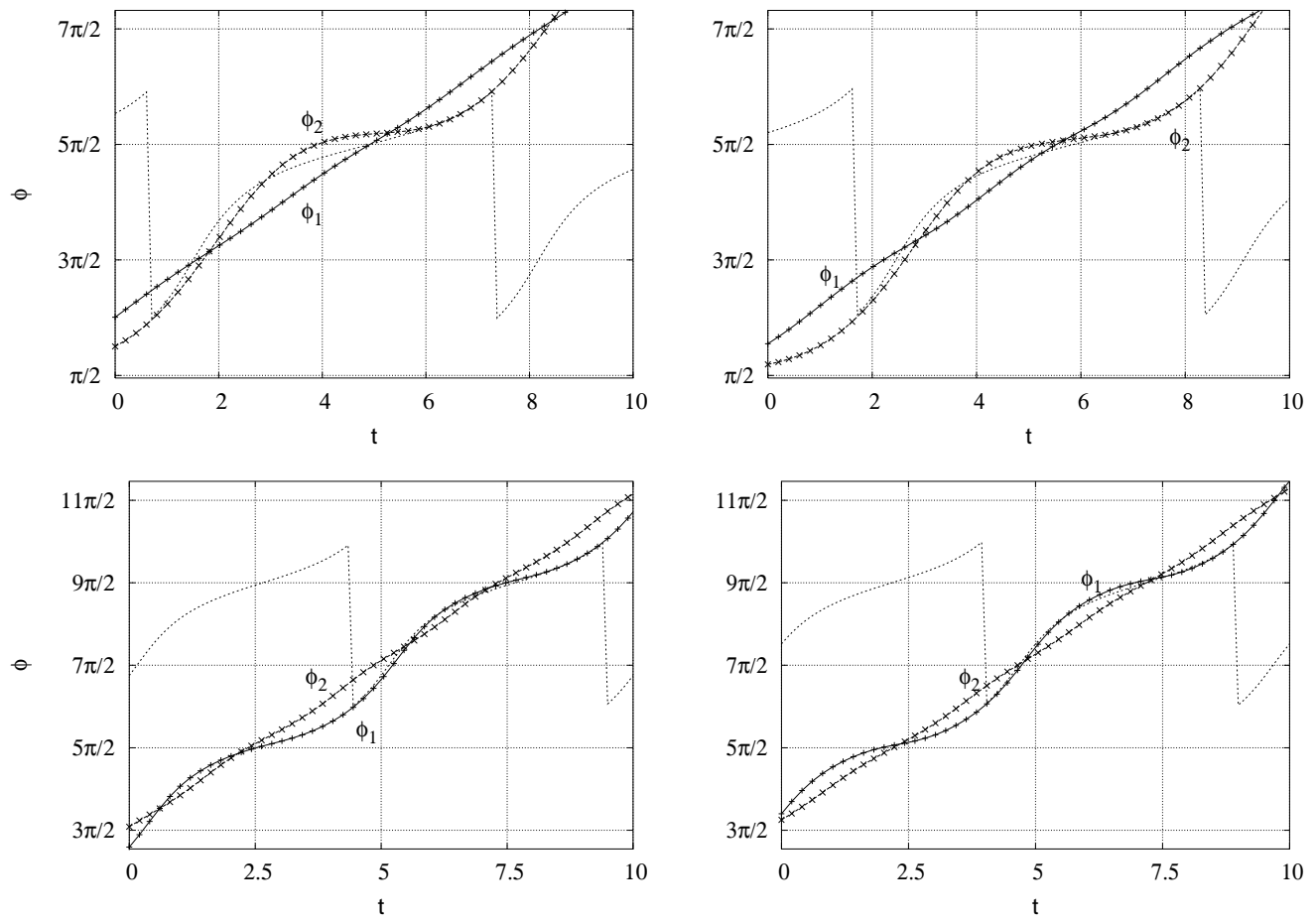

Figure 10: Plots of the phases at the junctions $\phi\left(a_{1}, t\right)$ and $\phi\left(a_{2}, t\right)$ as a function of time for the IV curves in the two left panels of Fig. 9 The left panels are for $d_{1}=0.7, d_{2}=0.3$ and the right panels for $d_{1}=d_{2}=0.5$. The top panels show the resonance of order 3 while the bottom panels show the resonance of order 4 . Note how the junctions switch between ohmic mode and junction mode. The analytic expressions for the corresponding junction modes (35) are shown in dashed line. 

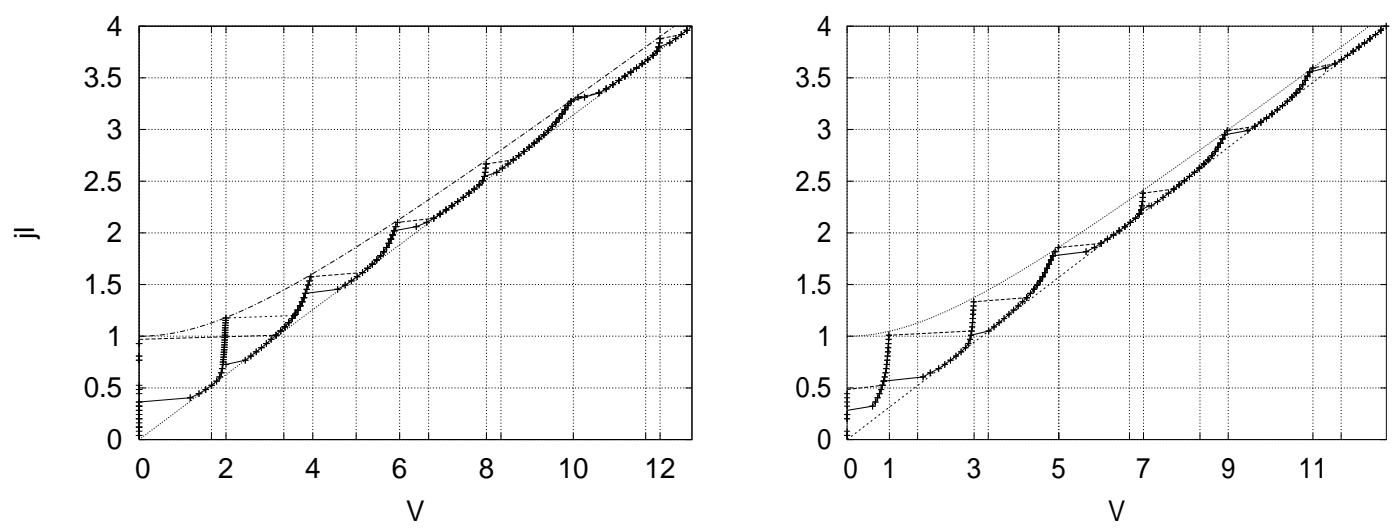

Figure 11: IV curves for two symmetrically placed junctions $a_{1}=2$ et $a_{2}=$ 8.The scale on the $x$ axis is in units of $\frac{\pi}{l}$. In the left panel the junctions are identical while in the right panel the second one is a $\pi$-junction. Note the resonance on the even harmonics $\frac{2 k \pi}{l}$ in the left panel and on the odd harmonics $\frac{(2 k+1) \pi}{l}$ in the right panel. We could have obtained the same change of IV curves for the same array of standard junctions by an appropriate choice of the magnetic field (see text). 